\section{Refining the ever-evolving molecular landscape of spitzoid melanocytic neoplasms}

DOI: $10.1111 /$ bjd. 17115

Linked Article: Farah et al. Br J Dermatol 2019; 180:404-408.

In this issue of the BJD, Farah et al. reported a case of spitzoid melanoma with morphological characteristics of an ALKrearranged spitzoid neoplasm and copy number gain, rather than rearrangement, of the ALK gene. ${ }^{1}$ This case is interesting in that it demonstrates a novel mechanism by which ALK may be activated in spitzoid melanoma. It also expands the spectrum of ALK alterations associated with the plexiform spitzoid phenotype, namely intersecting fascicles of amelanotic fusiform melanocytes. The authors additionally provided a concise overview of the different types of ALK alterations that have been identified in spitzoid neoplasms. In this modern era, where molecular data continue to accumulate at a fast pace, this article serves as a timely and up-to-date guide to this subject.

It is now known that approximately $50 \%$ of spitzoid lesions harbour kinase fusions of ALK, BRAF, ROS1, RET, NTRK1 or MET. ${ }^{2}$ Such fusions result in constitutive activation of oncogenic signalling pathways and ultimately proliferation of melanocytes. Of these kinases, BRAF is the most extensively studied in the realm of melanocytic lesions, and myriad activating alterations (point mutations, amplifications and gene rearrangements) have been identified. ${ }^{3}$ Our insight into ALK alterations in melanocytic lesions is following a similar path, where various activation mechanisms such as copy number gain and alternative transcription initiation are being increasingly recognized after the initial description of gene rearrangement. ${ }^{1,4}$

Besides kinases, a number of other genes (HRAS, CDKN2A, BAP1 and TERT) are implicated in the pathogenesis of spitzoid neoplasms. ${ }^{5}$ Many of these genetic changes are associated with a unique histomorphology and/or immunophenotype which may be used as surrogates of these changes, thereby streamlining the diagnostic process. The case illustrated by Farah et al. is an excellent example in which a plexiform spitzoid morphology raised consideration of an ALK-rearranged spitzoid melanoma, and prompted ALK immunohistochemistry and fluorescence in situ hybridization, which led to the finding of ALK copy number gain. ${ }^{1}$ Other well-known genotype-phenotype correlations include BAP1-inactivated atypical Spitz naevus/tumour and HRAS mutation/amplification in desmoplastic Spitz naevus, both of which are typically associated with an indolent behaviour, in contrast to those with homozygous CDKN2A deletion or TERT promoter mutation. ${ }^{5}$

As our understanding of the molecular underpinnings of spitzoid neoplasms continues to evolve, additional genes of interest may emerge, possibly implicating new mechanisms of neoplastic transformation. Refining the different modes of genetic alteration will likely prove important in diagnostic classification, as well as predicting prognosis and response to targeted therapy.

\section{Acknowledgments}

The author would like to thank Dr Paul W. Harms (Departments of Pathology and Dermatology, University of Michigan, U.S.A.) for his critical review of this commentary.

\section{Conflicts of interest}

None to declare.

Departments of Pathology and Dermatology, M.P. CHAN

University of Michigan, Ann Arbor, MI,

U.S.A.

E-mail: mpchan@med.umich.edu

\section{References}

1 Farah M, Nagarajan P, Curry JL et al. Spitzoid melanoma with histopathological features of ALK gene rearrangement exhibiting ALK copy number gain: a novel mechanism of ALK activation in spitzoid neoplasia. Br J Dermatol 2019; 180:404-8.

2 Wiesner T, He J, Yelensky R et al. Kinase fusions are frequent in Spitz tumours and spitzoid melanomas. Nat Commun 2014; 5:3116.

3 Shi H, Moriceau G, Kong X et al. Melanoma whole-exome sequencing identifies ${ }^{\mathrm{V} 600 \mathrm{E}} \mathrm{B}-\mathrm{RAF}$ amplification-mediated acquired B-RAF inhibitor resistance. Nat Commun 2012; 3:724.

4 Busam KJ, Vilain RE, Lum T et al. Primary and metastatic cutaneous melanomas express ALK through alternative transcriptional initiation. Am J Surg Pathol 2016; 40:786-95.

5 Tetzlaff MT, Reuben A, Billings SD et al. Toward a moleculargenetic classification of spitzoid neoplasms. Clin Lab Med 2017; 37:431-48.

\section{Is the routine screening for hyperhomocysteinaemia recommended in patients with chronic plaque psoriasis?}

DOI: $10.1111 /$ bjd. 17357

Linked Article: Tsai et al. Br J Dermatol 2019; 180:382-389.

Tsai et al. performed an extensive meta-analysis of observational studies investigating serum homocysteine, folate and vitamin B12 levels in patients with psoriasis and in a control population. ${ }^{1}$ Among 990 initially identified, they selected 24 high-quality studies that were considered eligible to be included. The major finding of the study was that psoriasis is significantly associated with hyperhomocysteinaemia and folate deficiency but not with vitamin B12 deficiency. In particular, the odds ratio of hyperhomocysteinaemia in patients with psoriasis was $3 \cdot 48$ (95\% confidence interval $2 \cdot 08-5 \cdot 83$ ), 